\title{
Análisis de la utilidad de la vacuna Candid 1 en la prevención de la fiebre hemorrágica argentina en niños
}

\author{
María Rosa Feuillade ${ }^{1}$ y Delia A. Enría ${ }^{1}$
}

Forma de citar Feuillade MR, Enría DA. Análisis de la utilidad de la vacuna Candid 1 en la prevención de la fiebre hemorrágica argentina en niños. Rev Panam Salud Publica. 2005:18(2):100-6.

RESUMEN Objetivo. Determinar la estrategia más racional de vacunación con Candid 1 para prevenir la fiebre hemorrágica argentina (FHA) en los menores de 15 años que viven en el área endémica. Métodos. Para el análisis de la efectividad estimada se diseñó un modelo de árbol de decisión, con dos posibles opciones: vacunar a todos los menores del área endémica (vacunación ampliada) o vacunar solamente a los menores de 15 años con mayor riesgo (vacunación selectiva). Estas opciones se compararon con la alternativa de no vacunar. La evaluación se complementó con un análisis de sensibilidad para identificar los valores umbral de las variables críticas que podrían modificar la decisión tomada. Las probabilidades empleadas se tomaron de estudios clínicos y epidemiológicos previos.

Resultados. Según el modelo empleado, la estrategia de vacunación ampliada fue la mejor opción, con una utilidad total esperada de 9,99998 (siendo 10 el valor máximo posible). El análisis de sensibilidad demostró que la vacunación selectiva sería la estrategia de mayor utilidad si la incidencia en la población de bajo riesgo se reduce a menos de 3 por 1000000 habitantes o si la tasa de reacciones adversas graves a la vacuna asciende a más de 9 por 100000 habitantes. Ninguna variación de los parámetros empleados en el modelo respaldó la opción de no vacunar. Conclusiones. Con los parámetros de riesgo y de beneficio empleados, se recomienda vacunar con Candid 1 a todos los menores de 15 años que viven en el área endémica de FHA. El modelo propuesto puede adaptarse a las necesidades futuras y ayudar a tomar decisiones mediante la incorporación de los datos prospectivos de la vigilancia de la enfermedad. Estos resultados pueden usarse como base para estudios de costo y eficacia y para otros análisis cuantitativos.

Palabras clave Virus de Junin, fiebre hemorrágica americana, técnicas de apoyo para la decisión, vacunas virales.

La fiebre hemorrágica argentina (FHA) es una enfermedad viral grave que afecta específicamente a los pobla-

\footnotetext{
Instituto Nacional de Enfermedades Virales $\mathrm{Hu}-$ manas Dr. Julio Maiztegui, Pergamino, Argentina. La correspondencia debe dirigirse a María Rosa Feuillade, Instituto Nacional de Enfermedades Virales Humanas Dr. Julio Maiztegui, Monteagudo 2510, 2700 Pergamino, Argentina. Correo electrónico: mariarosafeuillade@yahoo.com.ar
}

dores de la pampa húmeda, que es la región agrícola y ganadera más rica de Argentina. Su agente etiológico, el virus Junín, se aisló por primera vez en 1958 y desde entonces se han documentado brotes anuales de esta enfermedad en áreas cada vez más extensas (1). Como consecuencia de esto, la FHA ha aumentado su impacto negativo en la salud y la economía argentinas.
El roedor Calomys musculinus es el reservorio principal del virus Junín. Aunque se desconoce el mecanismo exacto de la transmisión del virus al ser humano, hay indicios de que su principal vía de propagación es por aerosol. La infección directa entre personas es poco habitual. Según su patrón epidemiológico, la FHA es cuatro veces más frecuente en hombres que 
en mujeres y $90 \%$ de los casos se presentan en la población rural; aproximadamente $10 \%$ de las personas afectadas son menores de 15 años. La incidencia de esta enfermedad varía en dependencia del lugar y el tiempo, ya que su diseminación es focal y depende de la presencia y de la cantidad de roedores infectados (1).

El tratamiento de la FHA se basa en la transfusión de plasma con alto contenido de anticuerpos específicos contra el virus Junín. Este procedimiento puede reducir la mortalidad de 15-30\% a menos de $1 \%$ cuando se administra tempranamente, aunque presenta los riesgos propios de los hemoderivados y $10 \%$ de los pacientes tratados pueden desarrollar el síndrome neurológico tardío $(1,2)$.

Debido a que es prácticamente imposible eliminar todos los roedores que sirven de reservorio de este virus, los esfuerzos para reducir la incidencia de la FHA han estado dirigidos a la obtención de una vacuna. Como resultado de un proyecto de colaboración internacional ${ }^{2}$ desarrollado a partir de 1979 se obtuvo un clon atenuado del virus Junín que se utilizó como principio activo de la vacuna denominada Candid 1 (3-5).

Los ensayos clínicos de fases I, II y III realizados entre 1985 y 1990 demostraron que la vacuna Candid 1 es segura e inmunogénica y su eficacia en hombres de 15 a 65 años de edad fue de $95,5 \%(5,6)$. Estos resultados contribuyeron a que en 1991 se decidiera vacunar mediante un esquema de uso compasivo poblaciones de adultos con mayor riesgo de infección, seleccionadas por presentar una elevada incidencia de FHA en los cinco años previos a la vacunación. Hasta la fecha se han vacunado con Candid 1 más de 240 000 adultos de uno $u$ otro sexo y se han confirmado los resultados de los ensa-

\footnotetext{
2 Proyecto PNUD Arg/78/009, Fiebre Hemorrágica. En ese proyecto participaron el Ministerio de Salud de la Nación y el Instituto de Enfermedades Virales Humanas Dr. Julio I. Maiztegui (INEVH), ambos de Argentina, el Programa de las Naciones Unidas para el Desarrollo, la Organización Panamericana de la Salud y el Instituto de Enfermedades Infecciosas del Ejército de los Estados Unidos de América (USAMRIID).
}

yos clínicos anteriores. La eficacia estimada de la vacuna en el período 19922004 fue de $97,7 \%$, con un intervalo de confianza de 95\% (IC95\%) de 95,5 a $98,8 \%$. Además, se ha constatado mediante anticuerpos neutralizantes que la respuesta inmunitaria específica se mantiene hasta 13 años después de la vacunación en más de $90 \%$ de los vacunados (7).

Sin embargo, solo se cuenta con cantidades limitadas de esta vacuna debido a que no hay interés comercial en su producción (vacuna "huérfana"). Aunque según se desprende de algunos estudios experimentales, la vacuna Candid 1 podría utilizarse para prevenir la fiebre hemorrágica boliviana (5), su uso se limita a una región de Argentina en la que vive una población aproximada de 5000000 personas (8). El Instituto de Enfermedades Virales Humanas Dr. Julio I. Maiztegui (INEVH) ha asumido la producción de esta vacuna que aún se encuentra en fase de ensayo clínico en adultos.

Una vez que la vacuna Candid 1 sea aprobada para su uso en adultos, se deberá valorar su posible aplicación en niños. El objetivo del presente estudio fue determinar la estrategia más racional de vacunación con Candid 1 para prevenir la FHA en menores de 15 años que viven en el área endémica.

\section{MATERIALES Y MÉTODOS}

\section{Base conceptual}

Para evaluar el impacto que puede tener la vacunación con Candid 1 en la reducción de la incidencia de la FHA en menores de 15 años se utilizó la metodología de análisis de la efectividad conocida como análisis de decisión (9). Este método utiliza un modelo de árbol de decisión que sirve para comparar estrategias alternativas y permite evaluar las posibles alternativas y arribar a un escenario final al que le corresponde un valor numérico que representa la utilidad esperada. De esta forma se puede seleccionar la opción que aporta el mayor beneficio o utilidad esperada (10-12).

El análisis de decisión se complementó con un análisis de sensibilidad que permitió ponderar las probabilidades incorporadas al modelo para las que existían condiciones de incertidumbre.

\section{Estructuración del problema}

Como primer paso se evaluó si era conveniente vacunar a los niños. Para la evaluación del alcance y del efecto de la vacunación se tomó en cuenta su utilidad para la sociedad en su conjunto - desde el punto de vista de la salud pública - y no los riesgos y beneficios de un individuo en particular.

Para la construcción del árbol de decisión se tuvieron en cuenta dos posibles opciones: vacunar a todos los menores del área endémica (vacunación ampliada) o vacunar solamente a los menores de 15 años con mayor riesgo (vacunación selectiva). Estas opciones se compararon con la alternativa de no vacunar.

Se definió que los beneficios de la vacunación con Candid 1 podían manifestarse en 10 años, ya que según la persistencia de los anticuerpos neutralizantes contra el virus Junín, una sola dosis de Candid 1 protege eficazmente a los adultos durante más de 10 años $(5,7)$.

\section{Diseño del árbol de decisión}

Las tres estaciones principales de decisión fueron la vacunación selectiva, la vacunación ampliada y no vacunar. A partir de estas estaciones principales se ubicaron los distintos escenarios intermedios posibles o nodos de probabilidad hasta arribar a un escenario final.

La estimación de las probabilidades para cada uno de los eventos controlados por el azar se basó en los datos clínicos y epidemiológicos de estudios anteriores (13) y de publicaciones sobre el tema. Las variables empleadas fueron (figura 1):

- Población total en riesgo en el área con FHA endémica: 5000000 habitantes

- Población total de menores de 15 años: 1400000 habitantes (13) 
FIGURA 1. Árbol de decisión: probabilidades estimadas y utilidades esperadas

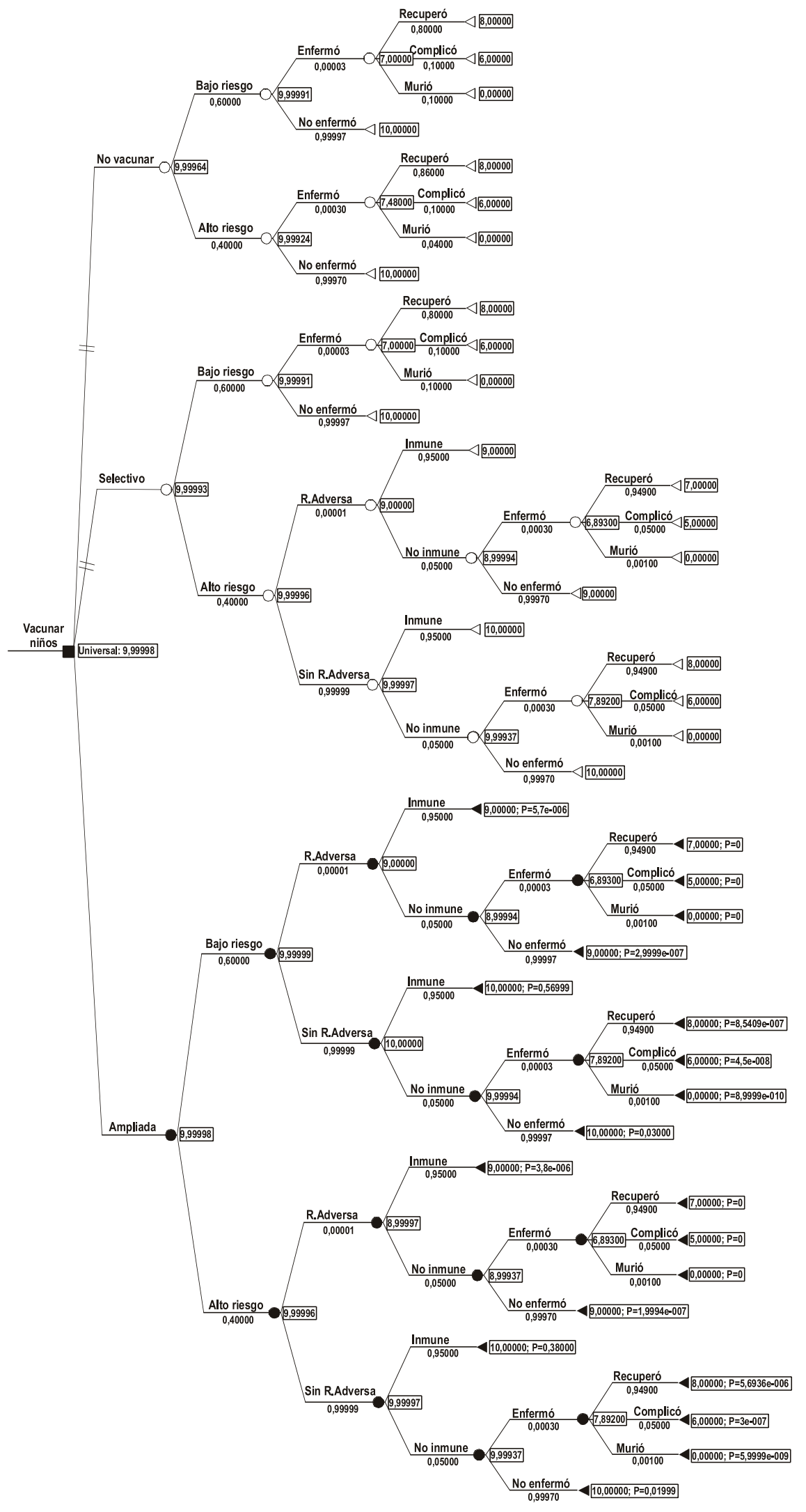

- Población de menores con alto riesgo: 587264 habitantes (13)

- Población de menores con bajo riesgo: 812736 habitantes (= $1400000-$ 587 264)

- Proporción de población con bajo riesgo (pBR): 0,60 (= $812736 /$ 1400 000)

- Proporción de población con alto riesgo (pAR): 0,40 (= $587264 /$ 1400 000)

- Probabilidad de enfermar en la población con bajo riesgo (pFHA1): 0,00003 . Este valor se calculó a partir de la incidencia en el período 1989-1998, estimada para el total de la población de menores de 15 años con bajo riesgo (= 21 / 812 736) (13).

- Probabilidad de enfermar en la población con alto riesgo (pFHA2): 0,0003 . Este valor se calculó a partir de la incidencia en el período 1989 1998, estimada para la población de menores de 15 años con alto riesgo (= 147 / 587 264) (13).

- Probabilidad de morir por FHA de las personas con bajo riesgo sin vacunar (pMU1): 0,10. Para definir este valor se tomó en cuenta la mortalidad promedio en el período 1991-2000 según los datos del Programa Nacional de Control de la FHA (14-21) y que la probabilidad de morir en los lugares con bajo riesgo es siempre mayor debido a que la población conoce menos la enfermedad y está menos alerta.

- Probabilidad de morir por FHA de las personas con alto riesgo sin vacunar (pMU2): 0,04. Este valor se estableció a partir de la tasa de mortalidad calculada para menores de 15 años en el período 1991-2000, que fue de 3,5\% (13).

- Probabilidad de morir de un vacunado que contrae la enfermedad (pMU3): 0,001. Para los propósitos de este modelo se consideró una probabilidad tan baja porque ninguno de los 240000 adultos vacunados ha muerto por FHA hasta el momento.

- Probabilidad de que se presenten complicaciones en personas sin vacunar enfermas con FHA, independientemente del riesgo (pCOM1): 0,10 . Esta valor se derivó de la tasa 
de presentación del síndrome neurológico tardío (10\%), que es la complicación más frecuente en los casos de FHA (1).

- Probabilidad de que se presenten complicaciones en personas vacunadas enfermas con FHA, independientemente del riesgo (pCOM2): 0,05 . Se estimó que esta probabilidad era menor que la de las personas sin vacunar, teniendo en cuenta que en los vacunados, la enfermedad se presenta en forma muy leve. Como el porcentaje de transfusión de plasma rico en anticuerpos específicos es menor, la tasa de complicaciones es también menor.

- Probabilidad de que se presenten reacciones adversas graves atribuibles a la vacunación con Candid 1 (pRA): 0,00001. Para este modelo se utilizó una estimación teórica de 1 por 100000 habitantes, ya que no se han informado efectos adversos graves en los adultos vacunados con Candid 1 en los últimos 10 años.

- Probabilidad de estar protegido contra la FHA gracias a la vacuna Candid 1 (pINM): 0,95. Este valor se tomó de la eficacia mostrada por la vacuna Candid 1 en hombres adultos en un estudio prospectivo, aleatorio, con doble enmascaramiento (6).

\section{Evaluación de las consecuencias}

Para esta etapa del análisis de decisión se creó una escala de valores de utilidad. Para ello, los resultados se ordenaron de mejor a peor y se asignaron valores entre 10 y 0 a las diferentes alternativas del árbol, de la siguiente manera:

- $\mathrm{uNO1}=10$, valor asignado a todos los escenarios que finalizaron sin enfermedad

- $\mathrm{uNO} 2=9$, valor asignado al vacunado que tuvo una reacción adversa

- $\mathrm{uR1}=8$, valor asignado a la persona sin vacunar o vacunada, que se enfermó de FHA, no tuvo reacciones adversas y se recuperó sin complicaciones

- $\mathrm{uR2}=7$, valor asignado a la persona vacunada que se enfermó de FHA y tuvo reacciones adversas, pero no sufrió complicaciones

- $\mathrm{uC1}=6$, valor asignado a la persona sin vacunar o vacunada, que se enfermó de FHA sin reacciones adversas y sufrió alguna complicación

- $\mathrm{uC2}=5$, valor asignado a la persona vacunada que se enfermó de FHA, tuvo reacciones adversas y sufrió alguna complicación

- $\mathrm{uM}=0$, valor asignado a todos los escenarios que finalizaron con la muerte

Para calcular la utilidad esperada de cada estrategia se sumaron los productos de las probabilidades de cada evento y de sus resultados (figura 1).

\section{Análisis de la sensibilidad}

El análisis de sensibilidad permitió evaluar la robustez del cálculo de los valores y utilidades y controlar el grado de incertidumbre del árbol de decisión, ya que mostró el efecto que tuvieron las variables empleadas en el modelo sobre la decisión tomada.

En la primera etapa, el análisis de sensibilidad se desarrolló con un criterio de clasificación para cada uno de los parámetros seleccionados, con el fin de determinar si los umbrales mínimos y máximos esperados para cada uno de ellos producían cambios que afectaran la decisión propuesta por el modelo. Con las variables que resultaron críticas en este análisis se realizó el segundo análisis de sensibilidad con dos criterios para comprobar si cambios simultáneos en los valores de ambas variables afectaban a la decisión final elegida por el modelo.

Para el desarrollo del modelo de decisión y el análisis de sensibilidad se utilizó el software Data 4.0 Tree Age for Health Care (22).

\section{RESULTADOS}

Según el análisis de decisión, la vacunación ampliada de todos los niños del área endémica resultó ser la estrategia de mejor utilidad (figura 1), mientras que las opciones de no vacunar y de vacunar selectivamente a la población de menores con mayor riesgo de enfermar de FHA se descartaron. Según el modelo empleado, la utilidad total esperada para la opción seleccionada fue de 9,99998.

El análisis de la sensibilidad demostró que esa decisión puede modificarse si varían la incidencia de la FHA en la población con bajo riesgo (pFHA1) y la tasa de reacciones adversas graves a la vacuna (pRA). El valor umbral a partir del cual la incidencia de la FHA en la población con bajo riesgo puede revertir la decisión de vacunar a todos los niños del área endémica fue de 3 por 1000000 habitantes (figura 2), mientras que una tasa de reacciones adversas graves a la vacuna mayor de 9 por 100000 habitantes también puede modificar esa decisión (figura 3). Según el modelo empleado, estas dos variables resultaron críticas para el cambio de decisión, por lo que se realizó un análisis de sensibilidad con dos criterios de clasificación. Como se puede ver en la figura 4, a medida que disminuye la incidencia en la población con bajo riesgo y aumenta la tasa de reacciones adversas, la opción estratégica del modelo se inclina a favor de la vacunación selectiva.

Según el análisis de sensibilidad aplicado al resto de las variables del modelo, ninguna influyó en la estrategia seleccionada. Ninguna variación en los parámetros empleados en el modelo respaldó la opción de no vacunar.

\section{DISCUSIÓN}

La FHA es un grave problema de salud en la región pampeana de Argentina. La disponibilidad de una vacuna segura, inmunogénica y eficaz, como Candid 1, abre nuevas posibilidades para el control de esta enfermedad.

Candid 1 es una vacuna huérfana debido a la falta de interés comercial. Su producción inicial se realizó en la década de 1990 en el Instituto Salk, en Swiftwater, Pensilvania, Estados Unidos de América. En el proyecto de colaboración que respaldó su desarrolló se previeron las dificultades que se presentarían para su producción, por 
FIGURA 2. Análisis de sensibilidad con un criterio de clasificación de la probabilidad de enfermar en la población con bajo riesgo (pFHA1)

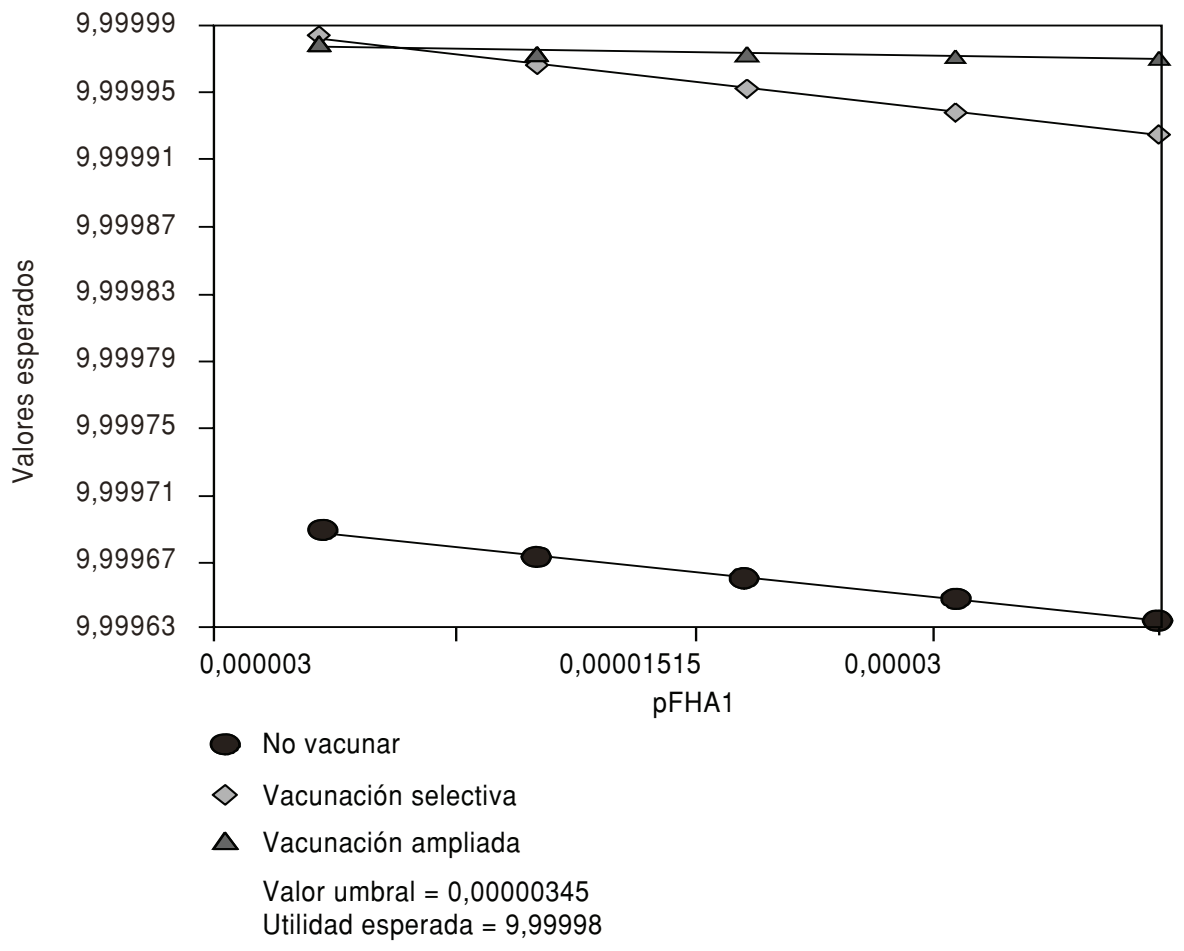

FIGURA 3. Análisis de sensibilidad con un criterio de clasificación de la probabilidad de presentar reacciones adversas graves atribuibles a la vacunación con Candid 1 (pRA)

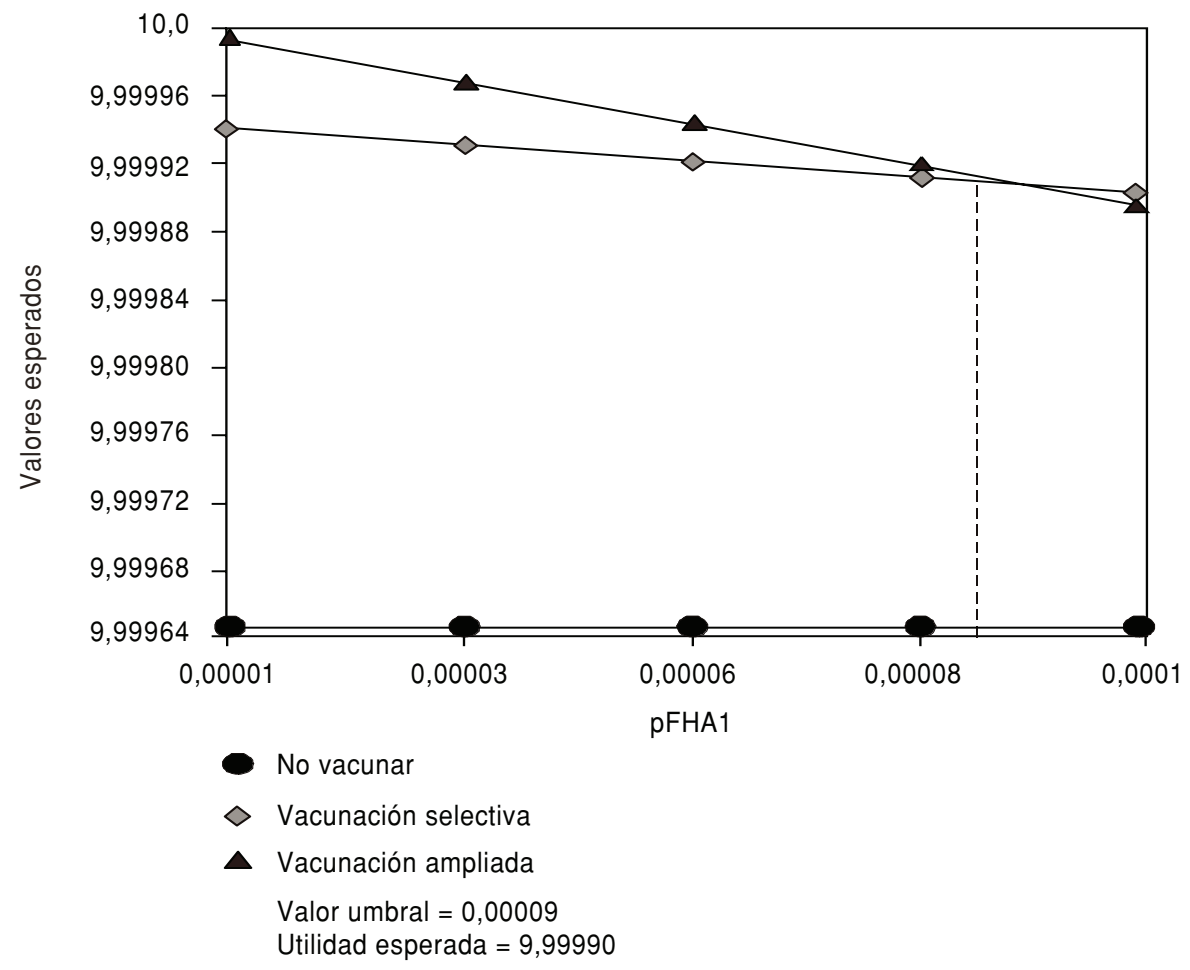

lo que se decidió que la vacuna se produciría en Argentina. En la década de 1990, el Ministerio de Salud retomó el proyecto de producción nacional y designó al INEVH como laboratorio productor. Hasta la fecha, el INEVH ha cumplido con los requisitos establecidos por las autoridades regulatorias para producir la vacuna Candid 1 y ya se cuenta con varios lotes de vacunas producidos en Argentina que se utilizarán para registrar esta vacuna en el país.

Aunque en un inicio se pensaba que el riesgo de adquirir FHA era bajo en menores y que esta enfermedad era menos grave en niños que en adultos, no existen estudios recientes que cuantifiquen ese riesgo y respalden esas aseveraciones $(23,24)$.

Entre las posibles estrategias para el control de la FHA está la vacunación de todos los menores de 15 años; sin embargo, no existe consenso al respecto en la comunidad científica. Los grupos que rechazan la estrategia de vacunación ampliada argumentan que el riesgo en niños es bajo, que la enfermedad es leve y que los riesgos que implica usar una vacuna con un virus vivo atenuado son mayores que los posibles beneficios de su aplicación masiva (25).

Sin embargo, se ha documentado que la vacunación selectiva de la población adulta con mayor riesgo de enfermar, principalmente los hombres, produjo un descenso significativo en la incidencia de la enfermedad. Además, se ha observado la modificación de los parámetros epidemiológicos característicos de esta enfermedad, entre ellos el aumento relativo del número de mujeres, de menores de 15 años y de habitantes urbanos y suburbanos que enferman de FHA (7).

En general, el análisis de decisión se realiza mediante la modelación de árboles de decisión que comparan las posibles estrategias. Según el presente estudio, la estrategia de vacunación ampliada - es decir, de vacunar a todos los niños del área endémica de FHA - ofrece mayores ventajas que la de vacunar selectivamente las poblaciones con mayor riesgo de padecer de FHA y que la de no vacunar. 
FIGURA 4. Análisis de sensibilidad con dos criterios de clasificación de la probabilidad de enfermar en la población con bajo riesgo (pFHA1) y de la probabilidad de que se presenten reacciones adversas graves atribuibles a la vacunación con Candid 1 (pRA)

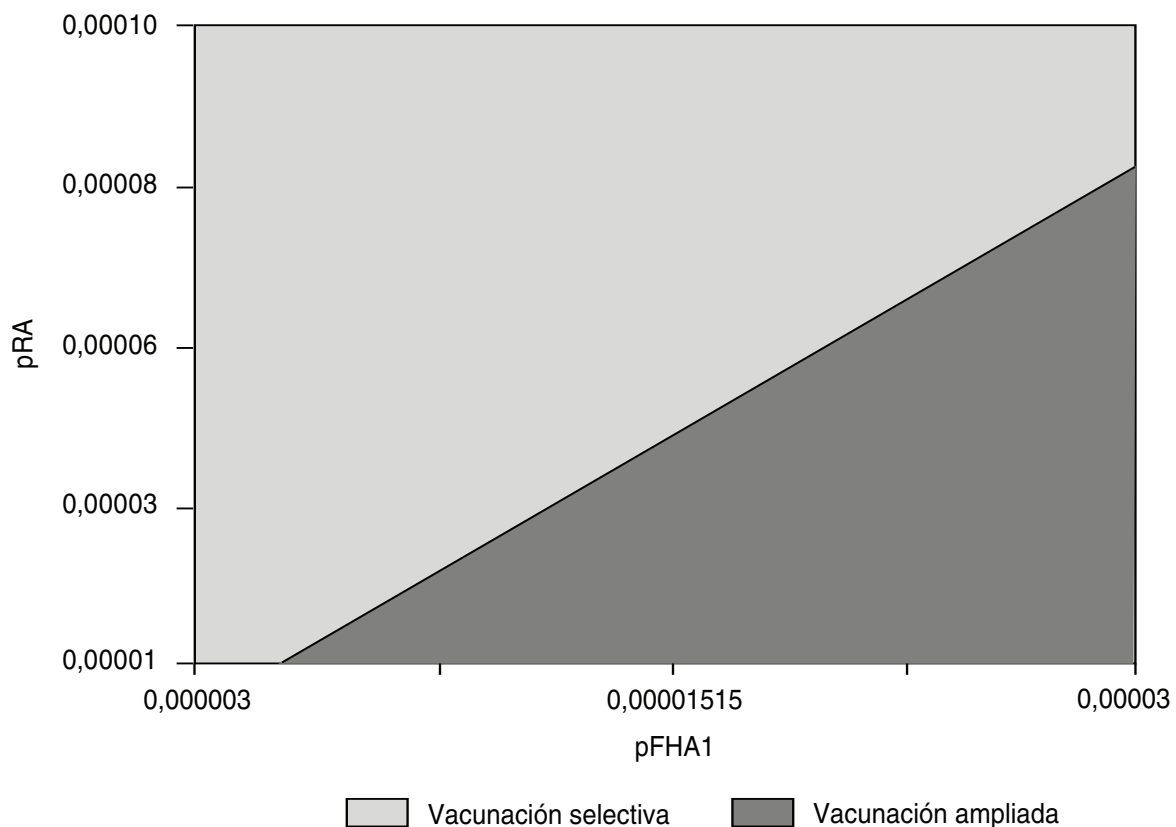

La estrategia de vacunación ampliada debe mantenerse mientras la incidencia de FHA en la población en bajo riesgo sea superior a 3 por 1000000 habitantes, de lo contrario se debe pasar a la estrategia de vacunar selectivamente a los menores en alto riesgo. $\mathrm{Si}$ la tasa de reacciones adversas graves de la vacuna llega a ser superior a 9 por 100000 personas, también se debe desestimar la estrategia de vacunación ampliada a favor de la vacunación selectiva. Es importante resaltar que ninguna variación en los parámetros em- pleados en el modelo respaldó la opción de no vacunar.

Los estudios de efectividad de la prevención proveen información básica que puede ayudar a corregir la marcha de los programas de salud pública y orientar pautas para la prevención y el control de la FHA, así como para la toma de decisiones en la asignación de recursos. El modelo propuesto en este estudio puede adaptarse a las necesidades futuras y ayudar a tomar diversas decisiones mediante la incorporación de datos prospectivos de la vigilancia de la enfermedad, como la eficacia de la vacuna y la proporción de reacciones adversas.

El modelo empleado no pretende seleccionar en esta etapa una estrategia de vacunación, sino ofrecer una evaluación inicial de las alternativas de vacunación de los niños del área endémica. Con los parámetros de riesgo y de beneficio empleados, el uso de la vacuna Candid 1 sería beneficioso para la comunidad, por lo que una vez que se autorice su aplicación en adultos, se deben emprender estudios clínicos en fase I y II en niños, según las normas reguladoras vigentes (26). Por otra parte, estos resultados pueden usarse como base para estudios de costo y efectividad y para otros análisis cuantitativos (27).

\section{REFERENCIAS}

1. Enría D, Briggiler AM, Feuillade MR. An overview of the ecological and preventive hallmarks of Argentine haemorrhagic fever (Junin virus). Bull Inst Pasteur. 1998;96:103-14.

2. Enría D, Maiztegui J. Antiviral treatment of Argentine hemorrhagic fever. Antiviral Res. 1994;23:23--31.

3. Barrera Oro JG, McKee KT. Toward a vaccine against Argentine hemorrhagic fever. Bull Pan Am Health Organ. 1991;25:118-26.

4. McKee KT, Barrera Oro JG, Kuehne AI, Spisso J, Mahlandt BG. Safety and immunogenicity of a live attenuated Junin (Argentine hemorrhagic fever) vaccine in Rhesus macaques. Am J Trop Med Hyg. 1993;48:403-11.

5. Enría DA, Barrera Oro J. Junin virus vaccines. En: Oldstone MBA, ed. Arenaviruses II. The molecular pathogenesis of arenavirus infections. Berlin: Springer Verlag; 2002. Pp. 239-61.
6. Maiztegui JI, McKee KT, Barrera Oro JG, Harrison L, Gibbs PH, Feuillade MR, et al. Protective efficacy of a live attenuated vaccine against Argentine hemorrhagic fever. J Infect Dis. 1998;177:277-83.

7. Enría D, Feuillade MR, Levis SC, Briggiler AM, Ambrosio AM, Saavedra MC, et al. Impact of the vaccination of high risk population for AHF with a live attenuated Junin virus vaccine. En: Saluzzo JF, Dodet B, eds. Factors in the emergence and control of rodent-borne viral diseases (hantaviruses and arenaviruses). París: Elsevier; 1999. Pp. 273-80.

8. Lang J, Wood S. Development of orphan vaccines: an industry perspective. Emerg Infect Dis J. 2000;5:749-56.

9. Teutsch S, Harris J. Introduction. En: Haddix A, Teutsch S, Shaffer P, Duñet D, eds. Prevention effectiveness. A guide to decision analy- sis and economic evaluation. New York: Oxford University Press; 1996. Pp. 3-9.

10. Snider DE, Holtgrave DR, Duñet DO. Decision analysis. En: Haddix AC, Teutsch S, Schaffer PA, Dunet DO, eds. Prevention effectiveness: a guide to decision analysis and economic evaluation. New York: Oxford, University Press; 1996. Pp. 27-45.

11. Miller M, Hinman A. Cost-benefit and costeffectiveness. Analysis of vaccine policy. En: Plotkin S, Orenstein W, eds. Vaccines. 3. ${ }^{\text {a }}$ ed. Philadelphia: WB Saunders; 1999. Pp. 107488.

12. Teutsch S, Haddix A. Decision analysis for public health. En: Haddix A, Teutsch S, Shaffer $P$, Duñet D, eds. Prevention effectiveness. A guide to decision analysis and economic evaluation. New York: Oxford University Press; 1996. Pp. 46-56. 
13. Feuillade M. Rol de la vacuna Candid 1 en la prevención de la fiebre hemorrágica Argentina en los niños [tesina de maestro en Salud Pública]. Rosario (Argentina): Instituto de la Salud Juan Lazarte, Centro de Estudios Interdisciplinarios, Universidad Nacional de Rosario; 2003.

14. Programa Nacional de Control de la Fiebre Hemorrágica Argentina. Informe de la reunión anual; marzo de 1994; Pergamino, Argentina. Buenos Aires: Programa Nacional de Control de la Fiebre Hemorrágica Argentina; 1994. Pp. 5-10.

15. Programa Nacional de Control de la Fiebre Hemorrágica Argentina. Informe de la reunión anual; junio de 1995; Villa María, Argentina. Buenos Aires: Programa Nacional de Control de la Fiebre Hemorrágica Argentina. Pp. 3-5.

16. Programa Nacional de Control de la Fiebre Hemorrágica Argentina. Informe de la reunión anual; agosto de 1996; Rosario, Argentina. Buenos Aires: Programa Nacional de Control de la Fiebre Hemorrágica Argentina; 1996. Pp. 3-5.

17. Programa Nacional de Control de la Fiebre Hemorrágica Argentina. Informe de la reunión anual; junio de 1997; Azul, Argentina. Buenos Aires: Programa Nacional de Control de la Fiebre Hemorrágica Argentina; 1997. Pp. 4-6.
18. Programa Nacional de Control de la Fiebre Hemorrágica Argentina. Informe de la reunión anual; junio de 1998; Córdoba, Argentina. Buenos Aires: Programa Nacional de Control de la Fiebre Hemorrágica Argentina; 1998. Pp. 4-6.

19. Programa Nacional de Control de la Fiebre Hemorrágica Argentina. Informe de la reunión anual; julio de 1999; Venado Tuerto, Argentina. Buenos Aires: Programa Nacional de Control de la Fiebre Hemorrágica Argentina; 1999. Pp. 4-6.

20. Programa Nacional de Control de la Fiebre Hemorrágica Argentina. Informe de la reunión anual; junio de 2000; Pergamino, Argentina. Buenos Aires: Programa Nacional de Control de la Fiebre Hemorrágica Argentina; 2000. Pp. 4-6.

21. Programa Nacional de Control de la Fiebre Hemorrágica Argentina. Informe de la reunión anual; junio de 2001; Río Cuarto, Argentina. Buenos Aires: Programa Nacional de Control de la Fiebre Hemorrágica Argentina; 2001. Pp. 5-7.

22. Tree Age Software. Data 4.0 for health care. Williamstown (Maryland): Tree Age Inc.; 2001.

23. Albuerne JC, de Albuerne MA, Fernández NJ, Bustos OJ, Schwarz ER, Maiztegui JI. Fiebre hemorrágica argentina en el niño. V Jornadas
Nacionales de Enfermedades Transmisibles; octubre de 1975; La Plata, Argentina; 1975.

24. Ruggiero $\mathrm{H}$, Magnoni $\mathrm{C}$, Nota $\mathrm{N}$, Milani $\mathrm{H}$, Pérez Izquierdo F, Maglio F, et al. Fiebre hemorrágica argentina. Buenos Aires: El Ateneo; 1982.

25. Sabattini M, Larghi O. Informe del grupo de trabajo sobre vacuna Candid 1. Rev Asoc Argentina Microbiol. 1996;121(noviembrediciembre):7-11.

26. World Health Organization. Bridging studies. WHO guidelines on clinical evaluation of vaccines: regulatory expectations. Geneva: WHO; 2004. (Technical Report Series No. 924, Annex 1).

27. Haddix A, Shaffer P. Cost-effectiveness analysis. En: Haddix A, Teutsch S, Shaffer P, Duñet $\mathrm{D}$, eds. Prevention effectiveness. A guide to decision analysis and economic evaluation. New York: Oxford University Press; 1996. Pp. 103-29.

Manuscrito recibido el 29 de septiembre de 2004. Aceptado para publicación, tras revisión, el 22 de marzo de 2005.

ABSTRACT Objective. To determine the most rational strategy of vaccination with Candid 1 vaccine in order to prevent Argentine hemorrhagic fever among children under 15 years old living an endemic area.

\section{Analysis of the usefulness of Candid 1 vaccine in preventing Argentine hemorrhagic fever in children}

Methods. To analyze the estimated effectiveness, a decision tree model was designed, with two possible options: vaccinate all the children under age 15 in the endemic area ("expanded vaccination") or vaccinate only the children at greater risk ("selective vaccination"). These two options were compared with the alternative of not vaccinating. The evaluation was complemented with a sensitivity analysis to identify the threshold values of the critical variables that could change the decision. The probabilities that were used were taken from earlier clinical and epidemiological trials.

Results. According to the model that we used, the expanded vaccination strategy was the best option, with a total expected utility of 9.99998 (out of a maximum possible 10.0). The sensitivity analysis showed that selective vaccination would be the best strategy if the incidence in the population with low risk drops to less than 3 per 1000000 population or if the rate of serious adverse reactions to the vaccine reaches more than 9 per 100000 inhabitants. No variation in the parameters used in the model supported the option of not vaccinating.

Conclusions. Given the risk and benefit parameters that we used, we recommend vaccinating with Candid 1 all the children under age 15 who live in the area endemic for Argentine hemorrhagic fever. The proposed model can be fitted to future needs, and it can help in decision-making by incorporating prospective disease surveillance data. These results can be used as a basis for cost and efficacy studies and for other quantitative analyses.

Keywords Junin virus; hemorrhagic fever, American; decision support techniques; viral vaccines. 